J3eA, Journal sur l'enseignement des sciences et technologies de l'information et des systèmes, Volume 4, Hors-Série 2, 17 (2005)

DOI : http://dx.doi.org/10.1051/bib-j3ea:2005717

(C) EDP Sciences, 2005

Vidéomodèles - Méthode de visualisation 3D des circuits électriques en fonctionnement

F. Gueuning

Unité Électronique

Institut Supérieur Industriel ECAM

14 rue du Tir

B-1060 Bruxelles, Belgique 


\title{
VIDÉOMODÈLES - MÉTHODE DE VISUALISATION 3D DES CIRCUITS ÉLECTRIQUES EN FONCTIONNEMENT
}

\author{
F. Gueuning, Unité Électronique, Institut Supérieur Industriel ECAM, 14, rue du Tir, \\ B-1060 Bruxelles. gng@ecam.be
}

\begin{abstract}
Résumé :
Une grande difficulté rencontrée lors de l'étude de l'électricité réside dans l'absence de vision du fonctionnement des circuits. Depuis quelques années, plusieurs travaux ont été menés pour tenter de représenter ce fonctionnement. Les vidéomodèles constituent une solution haut de gamme dans ce domaine. Il s'agit d'animations 3D montrant l'évolution des tensions et courants de façon quantitative et très visuelle grâce à une représentation élaborée des composants obtenue par images de synthèse. Une première prospection réalisée auprès de personnes de tous niveaux de formation en électricité ou en électronique a révélé que plus de quatre personnes sur cinq, quel que soit leur niveau, estiment que les vidéomodèles favorisent sensiblement leur perception du fonctionnement des circuits. On présente ici les fondements de la méthode et l'on définit les symboles des dipôles de la théorie des circuits linéaires, puis on donne un exemple basé sur un circuit résistif.
\end{abstract}

Mots clés : vidéomodèle, animation 3D, circuit, électrique, électronique, simulation, comportement dynamique, fonctionnement, vidéo, modèle 3D.

\section{INTRODUCTION}

L'objectif est une représentation de circuits en fonctionnement directement assimilable par notre cerveau si performant dans l'analyse d'images 3D dynamiques auxquelles il est habitué depuis la plus tendre enfance. Par cette représentation, on parvient à augmenter très sensiblement la rapidité de compréhension et de vision d'ensemble qui faisait cruellement défaut jusqu'à présent dans le monde de l'électricité et de l'électronique en particulier. Cette représentation conviviale est rigoureuse et quantitative, ce qui lui permet de convenir à la fois pour le débutant et pour le spécialiste, chacun pouvant y découvrir ce qui l'intéresse.

En effet, outre la vision du fonctionnement de l'ensemble, qui convient plutôt dans une première approche, on peut se focaliser dans un second temps sur les détails qui sont également présents dans le même vidéomodèle. On a baptisé de ce nom ce type d'animation [1] qui permet enfin de voir un circuit en pleine action (video: je vois) mais que l'on ne s'y trompe pas, ce que l'on voit n'est qu'une représentation, un modèle où la troisième dimension joue un rôle particulier encore méconnu de la plupart des électriciens.

\section{RÈGLES GÉNÉRALES}

Voyons tout d'abord quelles sont les grandeurs électriques qui seront représentées et comment.

\subsection{Représentation de 2 grandeurs électriques}

L'idée de base est de représenter au moins deux grandeurs électriques de nature différente directement à l'emplacement du circuit où elles interviennent (deux car le comportement est lié aux interactions de deux grandeurs). Il s'agit d'en prendre une parmi tension, potentiel et champ électrique, et l'autre parmi charge, courant et champ magnétique, ou encore de prendre impédance et puissance. Comme chaque composant d'un circuit exprime ses exigences le plus simplement en termes de tensions et courants, on a décidé de se focaliser sur ces deux grandeurs. Toutefois, plutôt que les tensions au bornes, on a choisi, comme d'autres [2], [3], [4], de représenter le potentiel en chaque noud du circuit, et ce à chaque instant, donc en fonction du temps.

\subsubsection{Potentiel et plan de masse}

Le potentiel en chaque nœud du circuit est représenté par la troisième dimension d'un espace tridimensionnel (les deux premières constituant le dessin classique du circuit). Lorsque le circuit évolue en fonction du temps, le potentiel de chaque nœud peut varier et déplacer en conséquence la coordonnée $Z$ correspondante [1], [2], [3], [4].

Afin de disposer d'un plan de repère dans cette représentation à trois dimensions, le circuit apparaît en filigrane à deux dimensions dans le plan horizontal de masse (donc à 0 volt). 


\subsubsection{Courant}

Pour représenter le courant en sens et amplitude, différentes méthodes ont été utilisées sans être vraiment convaincantes [2], [4]. Pour les vidéomodèles [1], on a imaginé de le représenter par un cône entourant le conducteur, d'amplitude et longueur fonction de l'intensité et de sens correspondant au sens conventionnel à chaque instant. L'amplitude du cône est proportionnelle soit au courant I, soit à $\mathrm{I}^{0.4}$ lorsque les courants sont fort différents d'une branche à l'autre, comme dans les circuits électroniques. De couleur jaune, le cône possède également une bague bleue à l'avant pour que le sens reste visible même pour des courants faibles. De plus (fig 1), lorsque la valeur absolue du courant instantané est en augmentation, ce cône est gonflé à la façon d'une ogive (convexe) tandis que si la valeur est en diminution, le cône prend plutôt la forme d'un pavillon de trompette (concave). Ceci permet, lorsque l'on s'intéresse aux détails, de voir si le courant est en augmentation ou en diminution, y compris lors d'un arrêt sur image. Dans une bobine, par exemple, l'interaction entre variation du courant et tension induite est ainsi mieux perçue.

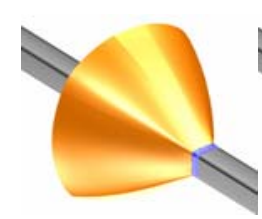

croissant

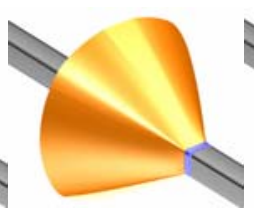

constant

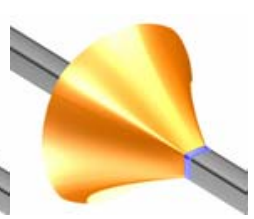

décroissant

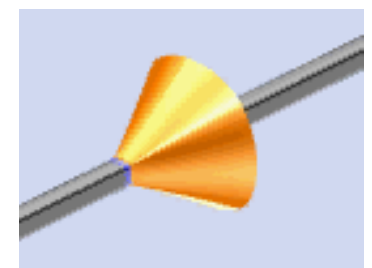

fig 1 : Représentation du courant

Pour ne pas encombrer inutilement le dessin, le courant n'est représenté que là où on le souhaite. Ce choix est opéré avant la fabrication du vidéomodèle.

\subsection{Représentation des conducteurs}

Les conducteurs, électriquement parfaits, apparaissent horizontaux. Contrairement à [2], [3], [4] où conducteurs et composants sont dessinés par de simples lignes, les conducteurs ont ici une section à peu près carrée pour renforcer la perception des plans équipotentiels. De cette manière, le regard prolonge leur face horizontale pour imaginer le plan équipotentiel. De plus, leur section est arrondie à la façon des bords des anciens écrans de télévision, ce qui permet d'obtenir directement un aspect de grande qualité lors du calcul des images de synthèse.

\begin{tabular}{|c|c|}
\hline Rôle du conducteur & Couleur suggérée \\
\hline non spécifique & gris \\
\hline masse & noir \\
\hline alimentation positive & rouge-gris \\
\hline alimentation négative & bleu-gris \\
\hline entrée ou sortie & doré \\
\hline terre & vert $\&$ jaune \\
\hline
\end{tabular}

table 1 : couleurs suggérées pour les conducteurs

Par défaut, les conducteurs sont de couleur grise. On suggère quelques conventions de couleurs non limitatives reprises à la table 1 .

\subsection{Symboles 3D des composants}

Contrairement aux dessins par lignes rencontrés ailleurs, on utilise ici un graphisme sophistiqué afin qu'il convienne même pour des circuits comportant jusqu'à plusieurs dizaines de composants. Il faut donc que, même dessinés avec un nombre réduit de pixels, les symboles et leur position dans l'espace soient aisément reconnus. Chaque composant est d'épaisseur limitée et apparaît assez plat lorsqu'il n'est soumis à aucune tension. De plus, 
sa forme 3D est judicieusement étudiée avec arrondis pour que les jeux de lumière calculés lors de la réalisation des images de synthèse le rendent identifiable au premier regard. Sa couleur aussi lui est spécifique.

\subsubsection{Dimensions XY des symboles}

Pour représenter les composants, faut-il faire intervenir leur taille réelle ou encore la valeur de leur paramètre principal? Même si pour les premiers pas d'un débutant il peut être intéressant que le dessin rende compte de la valeur d'un élément (ex: résistance), plusieurs raisons amènent à penser qu'une taille standard est préférable:

- Les symboles traditionnels de composants ne comportent pas d'information dimensionnelle. Il vaut mieux que dès le départ, le débutant s'habitue à cela et puisse directement établir un lien entre le schéma traditionnel et le vidéomodèle.

- Le repérage de la position dans un espace 3D est plus aisé si la taille de l'élément est simplement liée à son éloignement.

- Dans un circuit complexe, l'usage de symboles de tailles différentes risque de nuire à la lisibilité. Toutefois, cette hypothèse n'a pas été testée et il n'est pas impossible que l'on constate dans le futur que des tailles différentes s'avèrent intéressantes dans certains cas.

\subsubsection{Déformation subie par les composants lorsque les potentiels évoluent}

On constate que traditionnellement dans un schéma, chaque composant est représenté par un symbole sans rapport avec sa constitution géométrique réelle. Seules les bornes d'accès sont parfaitement définies électriquement. On ne s'intéresse pas à ce qui se passe à l'intérieur des composants, en particulier à la façon dont le potentiel s'y répartit. Dans [1], [2], [3], [4], lorsqu'il existe une tension non nulle entre deux bornes d'un composant, leur coordonnée V ( $3^{\mathrm{e}}$ dimension) est différente et il convient de passer progressivement de l'une à l'autre. Dans la plupart des cas, il a été décidé d'adopter simplement une progression linéaire qui ne comporte aucune signification particulière.

\section{REPRÉSENTATION DES DIPÔLES DE LA THÉORIE DES CIRCUITS LINÉAIRES. \\ 3.1. Résistance}

On sait que la résistance se caractérise par l'équation $\mathrm{V}=\mathrm{R}$.I où la valeur de $\mathrm{R}$ est constante si l'élément est linéaire. Le symbole traditionnel est un zigzag [2], [3] ou un rectangle [4]. On a estimé [1] qu'un symbole simple suffisait pour cet élément si simple. On a donc adopté un parallélépipède rectangle dans le cas d'une tension nulle. Celui-ci voit la composante $\mathrm{V}$ de chacun de ses points se modifier d'image en image au gré de l'évolution des potentiels. Comme dit plus haut, seules les bornes ont leur composante V significative. La figure 2 représente une résistance connectée soumise à une tension.

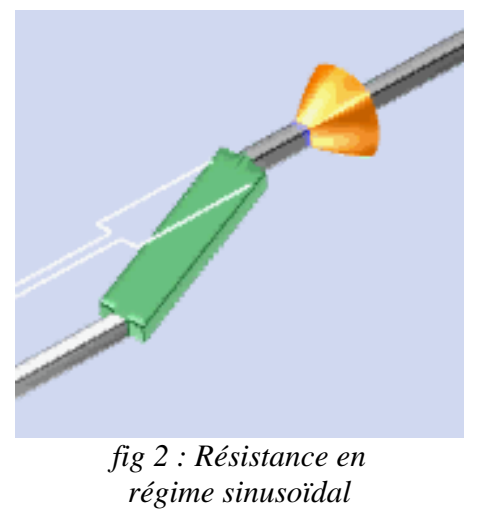

On aperçoit également la projection de ce fragment de circuit en "filigrane" sur le plan de masse, ce qui permet de déduire qu'une borne de la résistance est à la masse tandis que l'autre est à un potentiel positif. On a choisi la couleur verte pour la résistance. Le même symbole est utilisé pour une impédance ou une charge quelconque. C'est alors plutôt la couleur qui permet d'indiquer la nature résistive, capacitive, inductive (ou autre) de la charge.

\subsection{Capacité et condensateur}


La capacité se caractérise par l'équation $\mathrm{I}=\mathrm{C} . \mathrm{dV} / \mathrm{dt}$ où $\mathrm{C}$ est constante si l'élément est linéaire. Le symbole adopté (fig 3) est constitué de deux barres horizontales parallèles de couleur vert-bleu jointes par un faisceau de lignes droites discontinues parallèles dites lignes de champ électrique.

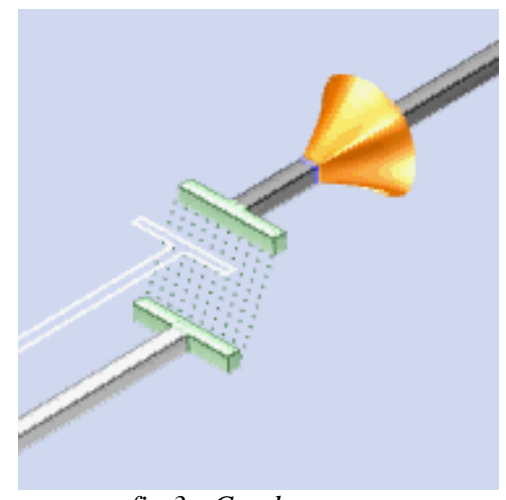

fig 3 : Condensateur

en régime sinusoïdal

Ces lignes de champ ne sont que symboliques. Le même symbole pouvant représenter n'importe quel condensateur, qu'il se réduise à une capacité pure ou soit un composant réel, quelles que soient ses dimensions, il ne faut voir dans la pente des lignes de champ qu'une information qualitative qui ne rend pas compte du champ électrique réel présent dans le composant.

\subsection{Inductance et bobine}

L'inductance répond à l'équation $\mathrm{V}=\mathrm{L} . \mathrm{dI} / \mathrm{dt}$ où $\mathrm{L}$ est constante dans le cas linéaire. Pour l'inductance pure de la théorie des circuits comme pour la bobine réelle, le symbole adopté, de couleur cuivre, est donné à la figure 4.

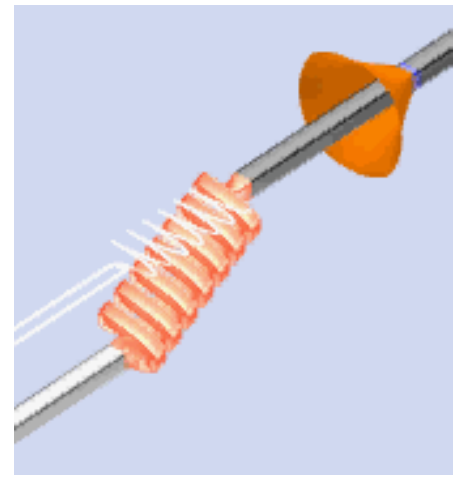

fig 4 : Inductance

en régime sinusoïdal

\subsection{Source de tension et source de courant}

Le symbole général de la source de tension idéale (continue, alternative ou quelconque) est donné à la figure 5. Il s'agit d'un disque de couleur rouge sombre déformé linéairement entre ses deux bornes. La source de courant, de couleur dorée, apparaît à la figure 6. Elle est constituée d'une flèche de courant entourée d'une ellipse. La flèche évolue de façon similaire aux cônes de courants décrits plus haut. 


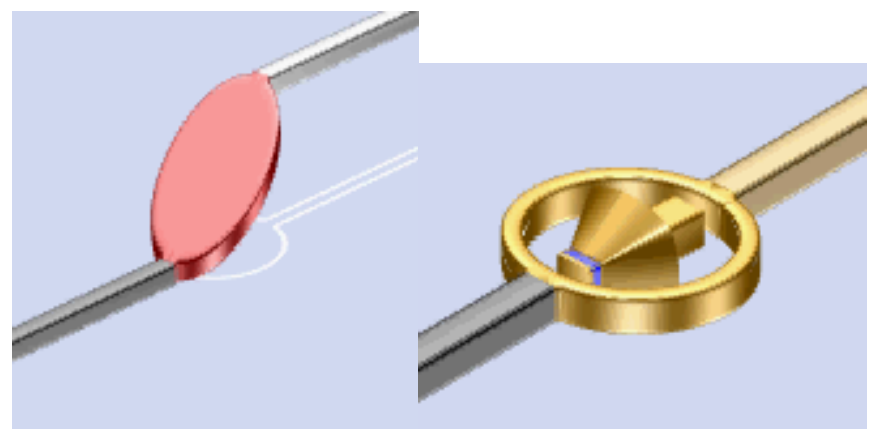

fig 5 : Source de tension

fig 6 : Source de courant

\section{EXEMPLE - CIRCUIT RÉSISTIF}

La figure 7 montre deux vues extraites d'un vidéomodèle de 100 images prévu pour tourner en boucle (fichier au format standard AVI de Microsoft). Le circuit représenté, comportant quatre résistances et une source de tension, est destiné à illustrer les lois de Kirchoff ainsi que la relation tension-courant dans les résistances linéaires.

Le dessin du haut de la figure correspond aux courbes de gauche où l'échantillon prélevé sur chaque signal apparaît au temps $7.2 \mathrm{~ms}$. Les valeurs numériques correspondantes sont données à droites des courbes. Pour chaque signal, on donne la valeur de l'échantillon utilisée pour produire le dessin 3D ainsi que la valeur de crête (peak) et la valeur efficace. Ces deux dernières ont été calculées sur l'ensemble des points de la courbe et restent constantes d'une image à l'autre.

La partie de gauche de ce circuit (entre $\mathrm{V}_{\mathrm{B}}$ et GND) peut modéliser une prise de courant domestique (230V $50 \mathrm{~Hz}$ ) et les résistances en parallèle sont deux charges de respectivement $60 \Omega$ et $180 \Omega$.

La simple observation du vidéomodèle amène à (re)découvrir les lois de base. On voit par exemple que tous les cônes de courant d'une même branche sont de même amplitude et de sens cohérent, ou encore que les tensions sont identiques sur les deux résistances en parallèle.

Le vidéomodèle de ce circuit est en général compréhensible par un débutant ignorant tout de l'électricité, bien qu'il s'agisse d'un circuit alternatif. 

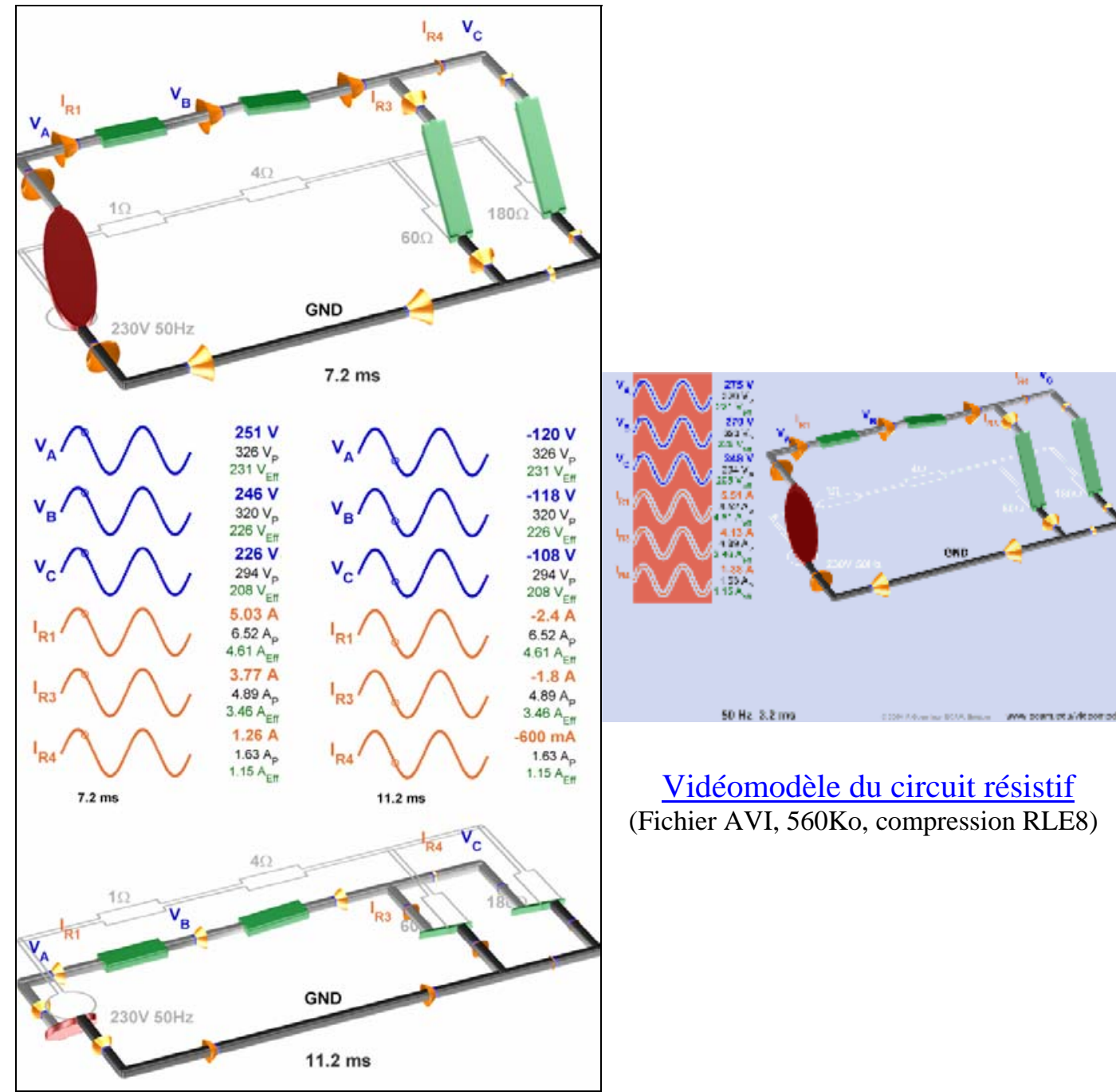

fig 7 : Deux vues d'un circuit résistif

\section{IMPORTANCE DE L'EFFET DYNAMIQUE}

Avec ce type d'animation, il devient plus simple de découvrir le fonctionnement des circuits en commençant par de l'alternatif plutôt que par du continu car le cerveau est bien plus rapide dans la perception dynamique des interactions que dans l'analyse d'images statiques où il est par contre plus précis. L'effet dynamique permet de donner rapidement une vue d'ensemble des interactions entre les composants.

Pour être efficace la fréquence de visualisation doit être comprise environ entre quelques dizièmes et quelques hertz. Dès lors, quelle que soit la fréquence du signal électrique observé, il convient évidemment de ramener son observation dans cette plage. Pour deux périodes de signal sur 100 images, une cadence de 15 images par seconde produit un effet visuel convenable. Des signaux de faible amplitude sont mieux décelés à fréquence plus élevée tandis que l'analyse de transitoires nécessite de ralentir l'allure.

\section{RÉALISATION}

La réalisation d'un vidéomodèle requiert plusieurs étapes : simulation SPICE, schéma 2D, cadrage, éclairage, choix du texte et des courbes à indiquer, fabrication proprement dite et validation du film obtenu. La fabrication des images de synthèse demande un temps calcul important, surtout pour un circuit complexe avec format d'image et degré de qualité conséquents : un film de 100 images en qualité haut de gamme (dite "auditoire") peut prendre quelques minutes à quelques dizaines de minutes.

\section{CONCLUSION}

Parmi les techniques de représentation quantitative des circuits en fonctionnement, les vidéomodèles se 
distinguent par la qualité du graphisme et de l'information indiquée. Il est ainsi possible de montrer des comportements sensiblement plus proches de la réalité, révélant bien plus d'effets de second ordre, que dans les autres méthodes puisque des images 3D de ce type, nourries de détails, ne dérangent pas le cerveau qui, dans la vie courante, en analyse en permanence en passant sans arrêt d'une vision d'ensemble à une vision de détail. Par exemple, la nuance entre représentations de courants croissant et décroisant ne dérange pas dans un premier temps mais permet une analyse plus fine par après. Cette qualité permet également la représentation de circuits relativement complexes. Dès lors, les vidéomodèles peuvent constituer un puissant outil non seulement pour les débutants, mais également pour les spécialistes, comme moyen de compréhension et d'expression.

Mais bien sûr, si l'on peut voir le fonctionnement des circuits, ce que l'on voit n'est qu'un modèle.

\section{Bibliographie}

1. ECAM, F. Gueuning : Méthode pour la visualisation, générée par ordinateur, de comportement d'assemblages de composants physiques, demande de brevet belge $n^{\circ}$ 2001/0619

2. Edward R. Doering, CircuitViz: A New Method for Visualizing the Dynamic Behavior of Electric Circuits, IEEE Trans. Educ., vol. 39, 1996

http://www.ece.msstate.edu/ hagler/Aug1996/005

/cd/index.htm

3. Charles R. Sullivan, Three-Dimensional Animations of Power-Electronics Circuits Visualize Voltage and Current, 2003

http://engineering.dartmouth.edu/other/3Dcircuits /background.html

4. A. del Río \& D. Valdés, Three-Dimensional Model for Analog Circuit Instruction, IEEE, 1997 http://www.ewh.ieee.org/soc/es/Nov1997/07 index.htm

5. L. Kleyman \& E. Skarbovsky, Animated Circuit Simulator, 2001

http://www.cs.technion.ac.il/ wagner/pub

/ckt_anim/docs/projectdoc.htm

6. site des vidéomodèles :

http://www.ecam.edu/videomodel 